\title{
Narrative review of the mechanisms of action of dachengqi decoction in the treatment of hyperlipidemic pancreatitis on six-hollow-organs to be unblocked theory
}

\author{
Guoxiong Liu ${ }^{1}$, Fang $\mathrm{Liu}^{2}$, Long Xiao ${ }^{3}$, Qiaogui Kuang ${ }^{4}$, Xingfeng He ${ }^{5}$, Yu Wang ${ }^{6}$, Yaming Wang \\ ${ }^{1}$ Emergency Department, ${ }^{2}$ Proctology Department, ${ }^{3}$ Department of Orthopedics, the First Affiliated Hospital of Guizhou University of TCM, \\ Guiyang, China; ${ }^{4}$ College of Acupuncture and Orthopedics, Guizhou University of Traditional Chinese Medicine, Guiyang, China; ${ }^{5}$ Graduate \\ School of Guizhou University of TCM, Guiyang, China; ${ }^{6}$ Department of Gastroenterology, ${ }^{7}$ Department of Science and Education, Puding County \\ Hospital of Traditional Chinese Medicine, Anshun, China \\ Contributions: (I) Conception and design: G Liu, F Liu; (II) Administrative support: L Xiao; Y Wang; (III) Provision of study materials or patients: Q \\ Kuang; (IV) Collection and assembly of data: L Xiao; Q Kuang; X He; Y Wang; (V) Data analysis and interpretation: G Liu, F Liu; (VI) Manuscript \\ writing: All authors; (VII) Final approval of manuscript: All authors. \\ Correspondence to: Fang Liu. Proctology Department, the First Affiliated Hospital of Guizhou University of TCM, Guiyang, China. \\ Email: liufang0013@126.com.
}

\begin{abstract}
The incidence rate of acute pancreatitis (AP) caused by hyperlipidemia is increasing year by year. The primary treatment goal is to reduce blood lipids rapidly. On the theory of "Six-hollow-organs to be unblocked" we used dachengqi decoction (original prescription of Zhang Zhonging in Shanghan Lun) to block the peroxisome proliferator-activated receptor $\gamma$ (PPARG) pathway and rapidly reduce blood lipid to achieve the purpose of treating hyperlipidemic acute pancreatitis (HLAP). In this review, we summarize the etiology and pathogenesis of HLAP and the progress of traditional Chinese medicine in treating HLAP. The mechanisms of action of dachengqi decoction in the treatment of HLAP and the involvement of the PPARG pathway were discussed. In brief, the dachengqi decoction has the effect of resolving phlegm and clearing waste substances and can improve intestinal function; can inhibits the production of interleukin-1, interleukin- 6 , and tumor necrosis factor- $\alpha$, and reduces the damage of SIRS to human body; also it improves the microcirculation system by inhibiting the production of inflammatory factors, reducing, or eliminating the damage to vascular endothelial cells and microvessels, and improving vascular permeability. The clarification of the mechanisms of action of the drug is conducive to the extensive clinical application of the classical formula.
\end{abstract}

Keywords: Hyperlipidemic acute pancreatitis (HLAP); triglyceride (TG); dachengqi decoction; PPARG pathway; mechanisms of action

Submitted May 19, 2020. Accepted for publication Jul 13, 2020.

doi: $10.21037 / \mathrm{apm}-20-1332$

View this article at: http://dx.doi.org/10.21037/apm-20-1332

\section{Introduction}

Hyperlipidemia (HL) refers to the high level of blood lipid, especially the rise of triglyceride (TG), which can lead to some significant diseases harmful to human health, including coronary heart disease, stroke and pancreatitis (1). $\mathrm{HL}$ is the most common inducing factor of acute pancreatitis (AP) except biliary tract disease and drinking. According to a survey on the incidence of AP in China,
HLAP accounts for $8.2-12.6 \%$ (2). With the change of lifestyle, the incidence of HLAP is increasing year by year (3). Its pathogenesis is still unclear, which may be related to the toxic effect of increased free fatty acids from triglycerides (TGs), decomposition on the pancreas itself, and microcirculation disorder, and calcium overload of the pancreas. Mechanisms involved in pathogenesis of HLAP have not been completely elucidated, oxidative stress is 
regarded as a major risk factor. The clinical manifestation includes persistent upper abdominal or left upper abdominal pain, abdominal distention, or vomiting. Studies showed that most of the patients with HLAP had mild conditions and were treated by non-surgical treatment. However, the effect was uncertain (4). The key to prevent recurrence is to reduce blood lipids, change diet structure, and lose weight rapidly (5).

The data from many clinical applications of dachengqi decoction and related studies showed that dachengqi decoction was a classic formula that could act on the PPARG pathway to reduce blood lipid rapidly. The emodin in dachengqi decoction could suppress PPARG and reduce the maturation of adipocyte. It could also suppress local inflammation. In this study, the mechanism of the action dachengqi decoction is discussed by search literature in PubMed published in English. We present the following article in accordance with the Narrative Review Checklist (available at http://dx.doi.org/10.21037/apm-20-1332).

\section{Western medicine's understanding of the pathogenesis of HLAP}

The pathogenesis of HLAP has not been recognized by modern medicine and related research.

\section{The damage caused by free fatty acids}

Under normal conditions, free fatty acid (FFA) from TG decomposition combines with serum albumin in vivo to form lipoprotein, which has no harm to pancreatic cells. When the increase of FFAs is more significant than the binding capacity of serum albumin, FFA accumulation in vivo will occur. Therefore, it will cause a rupture of the pancreatic acini and damage to vascular endothelial cells, directly leading to pancreatic ischemia and acidification of the internal environment. These events further promote the activation and release of pancreatic lipase, thus leading to $\mathrm{AP}(6-8)$.

\section{Microcirculation disturbance}

HL refers to the increase of total cholesterol (TC) or TG in plasma, in which TG exists in plasma in chylomicrons and very-low-density lipoprotein $(9,10)$. When the content of chylomicrons in the patient's body increases, a substantial number of FFAs are produced from TG decomposition and deposit in the capillaries of the pancreas, resulting in the blockage of capillaries and local ischemia of the pancreas. The increase of chylomicrons and FFAs cause the change of hemodynamics, the increase of blood viscosity and the formation of thrombus, leading to the local microcirculation obstacle, ischemia, and the aggravation of microcirculation obstacle of the pancreas, which finally causes the necrosis of the pancreatic tissue and pancreatitis $(11,12)$.

\section{Inflammatory mediators}

At present, it is generally believed the pathogenesis of HLAP is the lipid peroxidation reaction of the membranaceous structures of cell and organelle induced by the increase of blood lipid, which changes the signal transmission process of the membrane receptor, leading to the increase of $\mathrm{Ca}^{2+}$ level in the cell membrane and the activation of trypsin (13). The abnormal activation of pancreatin induces granulocytes to produce and release many inflammatory substances, of which pancreatin activated abnormally causes damage to the cells and tissues of the pancreas. It is an essential link in the pathogenesis of the disease. Also, the activation of target cells further results in the activation of cytokines and the release of inflammatory substances, which are the decisive factors of systemic inflammatory response syndrome and multiple organ dysfunction secondary to hyperlipidemic pancreatitis (14). It has been found that FFAs can cause pancreatic injury by inducing inflammation and then promoting the production of many cytokines and inflammatory substances (15).

\section{TCM understanding of the name of HLAP}

The "phlegm turbid," "vertigo," "blood stasis," and "chest arthralgia" recorded in traditional Chinese medicine are comparable to the clinical symptoms of hyperlipidemia at present. From the broad concept of treatment, HLAP can be classified into the syndromes mentioned above and treated (16). According to the clinical manifestations, etiology, and pathogenesis of HLAP, its mild symptoms can be observed in "epigastralgia," "abdominal pain," "vomiting" and other syndromes in traditional Chinese medicine, and the severe ones can be classified into "syndrome due to accumulation of evil in the chest, "syncope and adverse syndrome" and other syndromes (17).

\section{TCM understanding of etiology and pathogenesis of hyperlipidemic pancreatitis}

HL is believed to associate with dysfunction of liver, spleen, and kidney. Due to the factors including irregular diet, 
eating so much fatty or sweet food, emotional disorder, which affect the functions of viscera, Qi and blood, the syndromes of phlegm, dampness, blood stasis, will be resulted from the stagnation of Qi and blood combining with too much body fluid retention in the body. The disorder of water and dampness metabolism is the critical factor of HLAP (18). The principal TCM syndrome is superficial excessiveness but with fundamental insufficiency, leading to the stagnation of liver qi, stagnation of water due to spleen deficiency, and weakness of kidney Yang Qi. The pathogenesis of HLAP is mainly due to the increase of the burden of liver, spleen, and kidney caused by improper diet, overeating fatty and sweet food, excessive drinking, infectious diseases, and internal injury. In traditional Chinese medicine, the pathogenesis of HLAP is in the spleen, stomach, kidney, liver, gall, and many other viscera (19).

\section{Treatment of HLAP}

\section{Dachengqi decoction}

On the treatment idea of "no blockage, no pain" (20) and the theory of "six-hollow-organs to be unblocked, "we used dachengqi decoction, an important formula for dredging the hollow-organs. Rhubarb is the major drug which has the function of purging heat and defecation and clearing the gastrointestinal stasis. Glauber's salt can reduce hardness and dryness, and help rhubarb to dredge the hollow-organs (21). Magnolia officinalis and Fructus aurantii were used to promote Qi circulation in the body and remove fullness, to achieve the goal of "six-hollow-organs to be unblocked" and to strengthen further the dredging functions of rhubarb and Glauber's salt again. The treatment discharges the waste from the body and causes pathogenic factors to go away. In this study, we also explore the mechanisms of action of dachengqi decoction in the treatment of HLAP from the perspective of modern medicine.

\section{The mechanism of the lipid-lowering effect of rhubarb in dachengqi decoction through PPARG pathway}

PPARG is related to the occurrence of HL. PPARG gene is a crucial transcription factor in adipogenesis, and its correct expression is essential in mature adipocyte formation (22). PPARG plays a vital role in the differentiation of adipocytes, the expression of specific genes, the metabolism of fatty acids, and the regulation of insulin sensitivity. PPARG is a vital indicator gene of adipocyte differentiation and a determinant of fatty acid deposition in adipocytes and involved in regulating fatty acid metabolism-related network. It has been regarded as a target gene for the treatment of fatty acid metabolism disorder in the clinic. PPARG is also involved in the immune response of the body by influencing the signal pathway. The tumor necrosis factor receptor has a positive regulatory effect on PPARG (23). It is found that emodin can directly affect PPARG, inhibit the transcription and expression of PPARG, and reduce the formation of fat cells. It is also found that emodin can inhibit the expression of PPARG by affecting the activity of tumor necrosis factor through negative feedback regulation to regulate the formation of fat cells. It shows that emodin can regulate fat metabolism directly or indirectly (Figure 1). Also, it is found that many components of rhubarb can inhibit the insulin resistance and lipid metabolism disorder induced by inflammatory factors, reduces the deposition of free fatty acids in pancreatic capillaries, maintain the balance of fat metabolism in vivo, inhibit the inflammatory response, and play a role in the treatment of $\operatorname{HLAP}(24)$.

\section{Dachengqi decoction improves intestinal function}

Most of the blood lipids in the body come from the absorption in the intestine. Dachengqi decoction can increase the peristalsis of the intestine and reduce the time of lipid in the intestinal tract to excrete the lipid through defecation (25). It is found that rhubarb in dachengqi decoction has the effect of resolving phlegm and clearing waste substances. The anthraquinone derivatives contained in rhubarb can effectively reduce the level of total cholesterol and TG in the serum of the body by restoring the gastrointestinal mucosal barrier and enhancing the gastrointestinal peristalsis to reduce cholesterol absorption (26). In the application of dachengqi decoction, it was found that all the drugs in dachengqi decoction could improve the intestinal function. In the treatment of HLAP, one of the purposes is to restore the intestinal function. The increase of the peristalsis function of the intestinal tract is beneficial for diluting and removing toxins from the body so that the inflammatory status of the body can be rapidly improved (27). A study showed that hesperidin derivatives contained in Fructus aurantii could inhibit thiolase, reduce the release of chemical media, and improve the intestinal tension and peristalsis. Magnolia officinalis showed a significant antibacterial effect. The combined action of these drugs in dachengqi decoction could protect intestinal mucosa directly or indirectly and promote its functional recovery (28). Shen et al. (29) used dachengqi decoction in treating AP. They found dachengqi decoction, could antagonize 5-hydroxytryptamine 7 


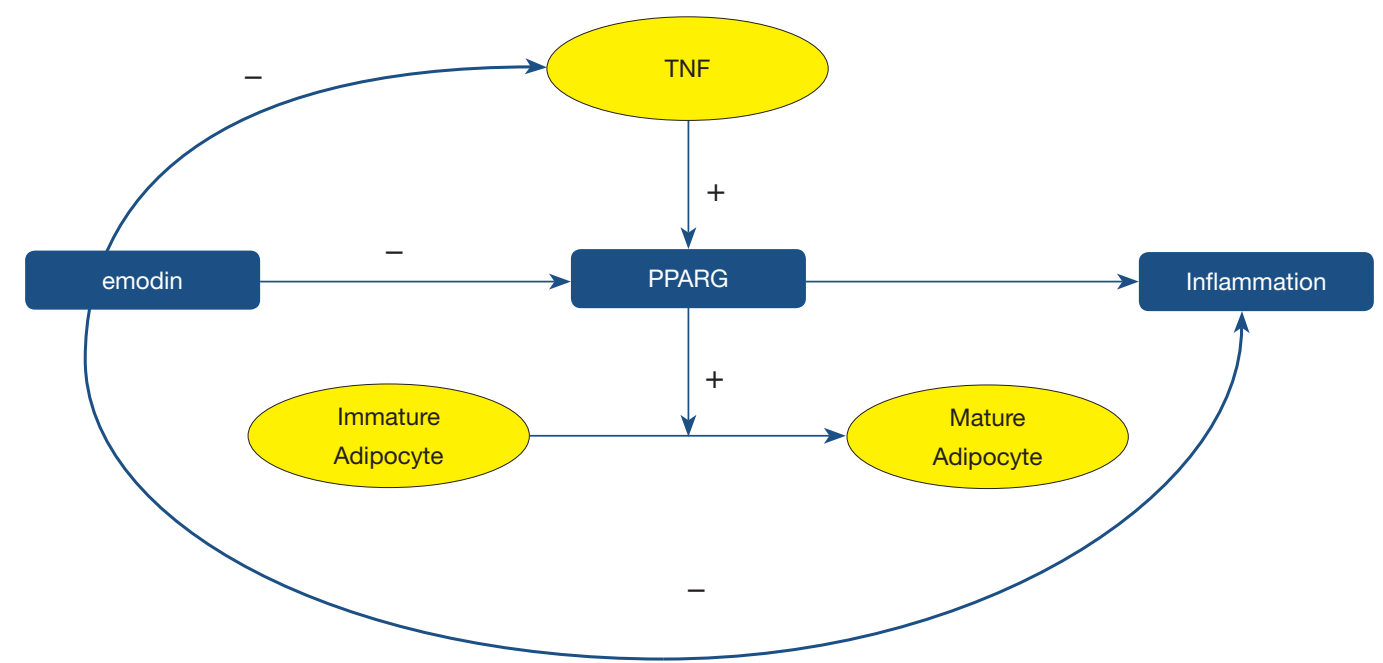

Figure 1 A brief mechanism of PPARG involved in fat metabolism. +, stimulate; -, inhibit.

receptors $(5-\mathrm{HT} 7 \mathrm{R})$ or reduce $5-\mathrm{HT}$ to produce indirectly inhibit to 5-HT7R function, relieve inhibition on intestinal nerves and relax intestinal circular muscles (30), thus to improve the peristalsis of intestinal circular muscles further, and restore the intestinal motility of pancreatitis patients. The treatment could repair and relieve the paralysis of the intestine caused by the damage and, in turn, recover the peristalsis function of the gastrointestinal tract. Yi et al. (31) found that dachengqi decoction could reduce the contents of diamine oxidase and endotoxin, which indicated that dachengqi decoction could restore the peristaltic function of the gastrointestinal tract. They also found that dachengqi decoction could inhibit the production of inflammatory factors, including tumor necrosis factor, interleukins, and hypersensitive C-reactive protein (hs-CRP) to eliminate the stimulation of inflammatory substances on the gastrointestinal tract and further improve gastrointestinal function. According to many modern medical types of research, no matter from a single drug or a compound preparation, dachengqi decoction can directly or indirectly improve the epithelial cells of the gastrointestinal mucosa and restore the gastrointestinal function.

\section{Dachengqi decoction inhibits inflammation}

The appropriate use of dachengqi decoction can effectively lower blood lipids and reduce the release of cytokines and inflammatory substances, thus to relieve inflammatory reaction in the pancreas before HLAP evolves into systematic inflammation reaction syndrome (SIRS). Among these factors, a considerable number of related features, including interleukin- 1 , tumor necrosis factor- $\alpha$, interleukin-6, are released, leading to the immune function injuries of the body. Dachengqi decoction inhibits the production of interleukin-1, interleukin- 6 , and tumor necrosis factor- $\alpha$, and reduces the damage to SIRS to multiple organs in the whole body in SIRS patients (32). Ying et al. (33) studied the effect of modified dachengqi decoction on serum inflammatory factors in rats with AP. They found that modified dachengqi decoction could reduce the production of amylase and lipase by inhibiting the expression of Nod-like receptor protein 3 (NLRP3), indirectly reduce the content of free fatty acids in the pancreas, reduce the inflammatory reaction in the pancreas, and then directly or indirectly reduce the production of inflammatory factors including interleukin-1 and interleukin-6. Zhang et al. (34) studied the effect of dachengqi decoction on the inflammatory factors in the upper digestive tract and found the treatment significantly lowered the levels of serum hsCRP, tumor necrosis factor- $\alpha$ and interleukin- 6 . In conclusion, dachengqi decoction and its single drug can inhibit the expression of cytokines, reduce the inflammatory substances in serum and pancreas, and play a role in protecting the pancreas. While inhibiting the expression of TNF- $\alpha$, it can indirectly affect PPARG and regulate blood lipids.

\section{Dachengqi decoction improves microcirculation}

In the pathogenesis of HLAP, the increase of chylomicrons and free fatty acids cause the change of hemodynamics, increasing blood viscosity and thrombosis. Rhubarb can inhibit platelet aggregation and adhesion, reduce 
blood coagulation factors, improve blood circulation in the pancreas, and promote the recovery of pancreatic microcirculation (35). A study revealed that Fructus aurantii could promote lipid metabolism, among which, Fructus aurantii flavone acted on Akt signaling pathway (36) through 3T3-L1 cells, thus inhibiting fat production. It could indirectly improve the microcirculation system of the pancreas by reducing chylomicrons and free fatty acids and decreasing the injury of vascular endothelium. Another study showed that dachengqi decoction could effectively inhibit the production of endotoxin, reduce or eliminate the damage to the intestinal epithelium, decrease the permeability of intestinal capillaries, improve local microcirculation and reduce the peroxide damage (37). Hu et al. (38) observed the effect of dachengqi decoction on the sepsis mice. They found it could eliminate damage factors, improve blood circulation, repair vascular endothelial cells, and microvessels.

Furthermore, it improves the microcirculation system by inhibiting the production of inflammatory factors, reducing, or eliminating the damage to vascular endothelial cells and microvessels, and improving vascular permeability. Zhou et al. (39) found that dachengqi decoction could increase gastrointestinal blood circulation, restore blood volume, reduce the release of inflammatory media, reduce the permeability of capillary epithelial cells and improve the microcirculation system. The above data show no matter from a single drug or a compound preparation, dachengqi decoction can improve the microcirculation disturbance and restore the blood microcirculation system.

\section{Conclusions}

The therapy of HLAP is improving on the current understanding of HLAP pathogenesis with the progress of medicine. To carry forward the advantages of traditional Chinese medicine, especially the classical prescriptions in Shanghan Lun in the treatment of HLAP, this paper summarizes and analyzes the clinical effect and the mechanism of action of dachengqi decoction and each drug in it in the treatment of HLAP discusses the effect of dachengqi decoction in lowering blood lipid and improving gastrointestinal function and analyzes the drug composition and action pathway in rapidly reducing blood lipid, improving microcirculation of pancreas and protecting endothelial cell structure from modern molecular medicine (40). We hope to make our contribution to promoting the progress of integrated traditional Chinese and Western medicine and the comprehensive development between traditional Chinese medicine and Western medicine.

\section{Acknowledgments}

Funding: Support project for scientific and technological research in traditional Chinese medicine and ethnic medicine, Guizhou Administration of Traditional Chinese Medicine. Project No.: QZYY-2019-092; Joint fund project of Guizhou Science and Technology Agency and Guiyang College of Traditional Chinese Medicine (Guizhou Science and Technology Agency), contract No.: Qiankehe LH [2015] No. 7787; Joint project fund of Guizhou Science and Technology Agency-hospital collaborative project, contract No: Qiankehe LH [2015] No. 7800; 2019 annual joint one health project of Guiyang Science and Technology Bureau and the First Affiliated Hospital of Guizhou University of Traditional Chinese Medicine, project No.: Zhuke contract [2019] 9-2-31.

\section{Footnote}

Reporting Checklist: The authors have completed the Narrative Review Checklist. Available at http://dx.doi. org/10.21037/apm-20-1332

Conflicts of Interest: All authors have completed the ICMJE uniform disclosure form (available at http://dx.doi. org/10.21037/apm-20-1332). The authors have no conflicts of interest to declare.

Ethical Statement: The authors are accountable for all aspects of the work in ensuring that questions related to the accuracy or integrity of any part of the work are appropriately investigated and resolved.

Open Access Statement: This is an Open Access article distributed in accordance with the Creative Commons Attribution-NonCommercial-NoDerivs 4.0 International License (CC BY-NC-ND 4.0), which permits the noncommercial replication and distribution of the article with the strict proviso that no changes or edits are made and the original work is properly cited (including links to both the formal publication through the relevant DOI and the license). See: https://creativecommons.org/licenses/by-nc-nd/4.0/.

\section{References}

1. Sun $\mathrm{H}, \mathrm{Zuo} \mathrm{HD}$, Lin Q, et al. MR imaging for acute 
pancreatitis: the current status of clinical applications. Ann Transl Med 2019;7:269.

2. Zhu Y, Pan X, Zeng H, et al. A Study on the Etiology, Severity, and Mortality of 3260 Patients With Acute Pancreatitis According to the Revised Atlanta Classification in Jiangxi, China Over an 8-Year Period. Pancreas 2017;46:504-9.

3. Zhang H, Zhou Z, Tan X, et al. Treatment and analysis of 69 patients with severe craniocerebral injury. Clinical Journal of Chinese Medicine 2013;(4):105-7.

4. Yin G, Cang X, Yu G, et al. Different Clinical Presentations of Hyperlipidemic Acute Pancreatitis: A Retrospective Study. Pancreas 2015;44:1105-10.

5. Glabay VP, Cridnev OV, Arkharov AV, et al. Complications of the 'open' surgeries for severe acute pancreatitis. Khirurgiia (Mosk) 2017;(10):72-6.

6. Song Y, Zhu H, Du Y. Advances in research of pathogenesis and treatment of hyperlipidemic acute pancreatitis. World Chinese Journal of Digestology 2019;27:112-6.

7. Wang G, Sun B, Jiang H. Research progress of hyperlipidemic acute pancreatitis. Chinese Journal of General Surgery 2005;14:777-9.

8. Sun Ci, Pan Y. Pathogenesis analysis and diagnosis and treatment progress in hyperlipidemic acute pancreatitis. World Chinese Journal of Digestology 2008;(4):343-9.

9. Song Y. Clinical study of the influence of serum triglyceride level on the prognosis of severe acute pancreatitis. Journal of Naval Medical University of the People's Liberation Army 2019.

10. Lin B. Effect of continuous hemofiltration on hyperlipidemia acute pancreatitis. Journal of Fujian Medical University 2014.

11. Wang Y, Sternfeld L, Yang F, et al. Enhanced susceptibility to pancreatitis in severe hypertriglyceridaemic lipoprotein lipase-deficient mice and agonist-like function of pancreatic lipase in pancreatic cells. Gut 2009;58:422-30.

12. Wang J. Clinical study on the treatment of obstructive sleep apnea hypopnea syndrome (phlegm-blood stasis syndrome) with modified Xuefu Zhuyu Decoction and Wendan Decoction. Journal of Chengdu University of Traditional Chinese Medicine 2017.

13. Gerasimenko JV, Gerasimenko OV, Petersen OH. The role of $\mathrm{Ca} 2+$ in the pathophysiology of pancreatitis. J Physiol 2014;592:269-80.

14. Liu D, Fu W, Li Q. Research progress on pathogenesis of hyperlipidemic acute pancreatitis. Modern Medicine \& Health 2015;23:3585-8.
15. Benítez S, Sanchez-Quesada JL, Lucero L, et al. Changes in low-density lipoprotein electronegativity and oxidizability after aerobic exercise are related to the increase in associated non-esterified fatty acids. Atherosclerosis 2002;160:223-32.

16. Tian Y, Pan L, Liu G. Overview of traditional Chinese medicine in the treatment of hyperlipidemia. Henan Traditional Chinese Medicine 2018;(9):1450-4.

17. Zhang S, Chen L. Application of Dachengqi Decoction in the treatment of acute pancreatitis. China Medical Herald 2009;31:70-1.

18. Wang Z, Chen P, Hong Y, et al. Research progress in the treatment of hyperlipidemia with Traditional Chinese Medicine. Journal of Practical Traditional Chinese Medicine 2019;(2):247-9.

19. Liu B, Wang Y, Liang W, et al. Clinical study on the treatment of hyperlipidemic acute pancreatitis with compound Dachaihu decoction combined with rhubarb external application. Journal of Community Medicine 2015;13:53-5.

20. Cao X. Clinical observation on the treatment of mild acute pancreatitis with modified Dachengqi Decoction combined with routine therapy. Hebei Journal of Traditional Chinese Medicine 2018;12:1858-61, 1868.

21. Du G. Clinical study on the treatment of severe acute pancreatitis with Dachengqi Decoction. Systems Medicine 2017;(6):106-9.

22. Stachecka J, Nowacka-Woszuk J, Kolodziejski PA, et al. The importance of the nuclear positioning of the PPARG gene for its expression during porcine in vitro adipogenesis. Chromosome Res 2019;27:271-84.

23. Min L, Chen D, Qu L, et al. Tumor necrosis factor-a polymorphisms and colorectal cancer risk: a meta-analysis. PLoS One 2014;9:e85187.

24. Du L, Yuan B, Zhang BX, et al. Study on Mechanism for Anti-Hyperlipidemia Efficacy of Rhubarb Through Assistant Analysis Systems for Acting Mechanisms of Traditional Chinese Medicine. Zhongguo Zhong Yao Za Zhi 2015;40:3703-8.

25. Liu Y. Forty cases of obesity and hyperlipidemia treated with Dachengqi Decoction combining with silkworm shit. Traditional Chinese Medicinal Research 2018;11:26-9.

26. Wang Z, Zeng Q, Wang J. Therapeutic effect of Jiangzhi Tongmai Decoction combined with rhubarb umbilical area applying on coronary heart disease with hyperlipidemia and its influence on serum Hcy, IL-6, APN and blood lipid index. Modern Journal of Integrated Traditional Chinese and Western Medicine 2019;14:1523-6. 
27. Shi M, Wu P. Effect of oral Dachengqi Decoction on laboratory indexes of patients with acute pancreatitis. Chinese Journal of Traditional Medical Science and Technology 2014;(6):629.

28. Ju Y, Zhang J, Zhu Z. Effect analysis of Dachengqi Decoction combined with propionyl glutamine on early severe acute pancreatitis. Shandong Journal of Traditional Chinese Medicine 2019;8:758-61.

29. Shen Y, Tang X, Jiang S, et al. Mechanism of Dachengqi Decoction in the treatment of intestinal dysfunction caused by severe acute pancreatitis. Chinese Archives of Traditional Chinese Medicine 2017;(5):1138-41, 1347-8.

30. Wang B, Mi D, Zhao F, et al. Research progress on the relationship between serotonin and slow transit constipation. Modern Journal of Integrated Traditional Chinese and Western Medicine 2016;35:3984-7.

31. Yi Q, Dai F, Guo Z, et al. Clinical observation on retention enema with modified Dachengqi Decoction in the treatment of 45 cases of severe acute pancreatitis complicated with acute respiratory distress syndrome. Journal of Traditional Chinese Medicine 2019;19:1657-62.

32. Feng X, Zhang S, Xiang R, et al. Clinical observation of hemoperfusion combined with hemodiafiltration in the treatment of hyperlipidemic acute pancreatitis. Journal of Sichuan Yixue 2017;(3):316-9.

33. Ying Y, Zhao J. Effect of Modified Dachengqi Decoction on serum inflammatory factors and apoptosis in rats with acute pancreatitis. Journal of Emergency in Traditional Chinese Medicine 2017;(7):1152-5.

34. Zhang J, Xie X. Effect of conventional western medicine

Cite this article as: Liu G, Liu F, Xiao L, Kuang Q, He X, Wang Y, Wang Y. Narrative review of the mechanisms of action of dachengqi decoction in the treatment of hyperlipidemic pancreatitis on six-hollow-organs to be unblocked theory. Ann Palliat Med 2020;9(4):2323-2329. doi: 10.21037/apm-20-1332 combined with modified Dachengqi Decoction on hs-CRP, TNF- $\alpha$ and IL- 6 in patients with early inflammatory ileus after upper gastrointestinal perforation. Modern Journal of Integrated Traditional Chinese and Western Medicine 2017;22:2443-5.

35. Dai J. Pharmacological research and clinical application of rhubarb. Tianjin Pharmacy 2011;(4):67-70.

36. Zhang X, Li Z, Ma Y, et al. Research progress on Fructus aurantii Immaturus. China Journal of Chinese Material Medica 2015;(2):185-90.

37. Chen H, Wu X, Guan F, et al. An experimental study on the protection of intestinal barrier function of Tongli Gunxia therapy in multiple organ dysfunction syndrome. Chinese Journal of Integrated Traditional and Western Medicine 2000;(2):41-3.

38. Hu X, Ni H, Liu K, et al. Effect of Dachengqi Decoction on pulmonary vascular permeability in septic mice. Journal of Emergency in Traditional Chinese Medicine 2016;(1):89-91.

39. Zhou L, He L. Mechanism of Dachengqi Decoction nasal feeding on gastrointestinal function recovery of ICU patients (gastrointestinal dryness-heat type). Journal of Sichuan of Traditional Chinese Medicine 2019;(3):120-2.

40. Yao D, Qin M, Cheng J. Observation on the effect of fenofibrate combining with low molecular weight heparin in the treatment of acute hyperlipidemic pancreatitis. Chinese and Foreign Medical Research 2018;16:162-4.

(English Language Editor: J. Chapnick) 